\title{
Age-Related Differences in Ventral Striatal and Default Mode Network Function During Reciprocated Trust
}

\author{
Dominic S. Fareri ${ }^{1}$, Katherine Hackett ${ }^{2}$, Lindsey J. Tepfer ${ }^{2}$, Victoria Kelly ${ }^{2}$, Nicole Henninger ${ }^{3}$, \\ Crystal Reeck ${ }^{4}$, Tania Giovannetti², David V. Smith ${ }^{2}$ \\ ${ }^{1}$ Gordon F. Derner School of Psychology, Adelphi University, Garden City, NY, USA \\ ${ }^{2}$ Department of Psychology, Temple University, Philadelphia, PA, USA \\ ${ }^{3}$ Lew Klein College of Media and Communication, Temple University, Philadelphia, PA, USA \\ ${ }^{4}$ Fox School of Business, Temple University, Philadelphia, PA, USA
}

\section{Manuscript information}

Number of pages: 31

Number of figures: 5

Number of tables: 1

Number of words for abstract: 229

Number of words for introduction: 758

Number of words for discussion: 1209

\section{Acknowledgments}

This work was supported, in part, by grants from the National Institutes of Health (R21MH113917, R03-DA046733, and RF1-AG067011 to DVS and R15-MH122927 to DSF) and a Pilot Grant from the Scientific Research Network on Decision Neuroscience and Aging [to DVS; Subaward of NIH R24-AG054355 (PI Gregory Samanez-Larkin)]. We thank Elizabeth Beard for assistance with task coding, Dennis Desalme, Ben Muzekari, Isaac Levy, Gemma Goldstein, and Srikar Katta for assistance with participant recruitment and data collection, and Jeffrey Dennison for assistance with data processing. DVS was a Research Fellow of the Public Policy $\mathrm{Lab}$ at Temple University during the preparation of this manuscript (2019-2020 academic year).

\section{Conflict of interest statement}

The authors have no conflicts to disclose.

\section{Data and code availability}

Data can be found on https://openneuro.org/datasets/ds003745. Analysis code can be found on https://github.com/DVS-Lab/srndna-trustgame. Thresholded and unthresholded statistical maps are located on https://identifiers.org/neurovault.collection:10447.

\section{Correspondence}

David V. Smith, Temple University

david.v.smith@temple.edu 


\section{Abstract}

Social relationships change across the lifespan as social networks narrow and motivational priorities shift to the present. Interestingly, aging is also associated with changes in executive function, including decision-making abilities, but it remains unclear how age-related changes in both domains interact to impact financial decisions involving other people. To study this problem, we recruited 50 human participants $\left(N_{\text {younger }}=26\right.$, ages $18-34 ; N_{\text {older }}=24$, ages $\left.63-80\right)$ to play an economic trust game as the investor with three partners (friend, stranger, and computer) who played the role of investee. Investors underwent functional magnetic resonance imaging (fMRI) during the trust game while investees were seated outside of the scanner. Building on our previous work with younger adults showing both enhanced striatal responses and altered default-mode network (DMN) connectivity as a function of social closeness during reciprocated trust, we predicted that these relations would exhibit age-related differences. We found that striatal responses to reciprocated trust from friends relative to strangers and computers were blunted in older adults relative to younger adults, thus supporting our primary pre-registered hypothesis regarding social closeness. We also found that older adults exhibited enhanced DMN connectivity with the temporoparietal junction (TPJ) during reciprocated trust from friends compared to computers while younger adults exhibited the opposite pattern. Taken together, these results advance our understanding of age-related differences in sensitivity to social closeness in the context of trusting others.

\section{Significance}

Our social relationships change as we grow older. Despite the ubiquity of these changes, little is known about how they impact our neural and behavioral responses to financial decisions involving other people. We examined this problem in the context of a trust game played with three partners varying in social closeness (friend, stranger, computer) while participants underwent fMRI. We found both blunted striatal activation and enhanced DMN-TPJ connectivity to reciprocated trust from friends relative to strangers and computers in older compared to younger adults. These results suggest that older adults demonstrate altered representations of social closeness within financial exchanges involving trust, which may expose older adults to increased risk for financial exploitation perpetrated by strangers. 


\section{Introduction}

The transition to older adulthood is dynamic, characterized by shrinking social networks, prioritization of social relationships and socially-centered goals, and an emphasis on positive relative to negative social experiences (Carstensen, 1992, 1995; Charles, 2010). These agerelated socioemotional changes are coupled with changes in neural networks supporting executive and social function (Andrews-Hanna et al., 2007; Devitt \& Schacter, 2020; Hughes et al., 2020; Laurita et al., 2020; Persson et al., 2006; Spreng et al., 2020), and taken together, such patterns have implications for older adults' abilities to successfully engage in social interactions. While an emerging body of literature has highlighted changes in decision-making in older relative to younger adults (Burr et al., 2021; Lighthall et al., 2018; Seaman et al., 2018) we know surprisingly little about how this translates to social decisions (i.e., trusting others). Extant findings suggest that older adults tend to perceive others as more trustworthy in general relative to younger adults and are less likely to pick up on subtle signs of dishonesty (Bailey et al., 2015, 2016, 2019; though see also Suzuki, 2018). Yet, work examining whether age-related differences emerge in decisions to trust close others, relative to strangers, and in the processing of reciprocity and betrayal is understudied and has implications for the vulnerability of older adults to financial exploitation.

Building and maintaining social relationships across the lifespan requires the ability to appraise someone as (un)trustworthy and effectively interpret their behavior within social interactions (Fareri, 2019). Research in young adults indicates that learning to trust draws on initial impressions of others that are dynamically updated with experienced patterns of reciprocity, a social reward signal (Chang et al., 2010); this process consistently recruits reward-related neural circuits (e.g., ventral striatum, medial/lateral prefrontal cortex; Bellucci et al., 2016; Fareri et al., 2012; Fouragnan et al., 2013). Moreover, our prior work (Fareri et al., 2015) demonstrates that these circuits differentially encode the value of reciprocity as a function of social closeness: reciprocity from friends relative to strangers elicits enhanced activation of the ventral striatum. This increased value signaling may in turn reinforce already close bonds, and in fact, decisions to trust in younger adults are strongly related to beliefs about safety (Chen et al., 2021). Yet, how social outcome processing shifts as we transition to older adulthood remains an outstanding question.

Critically, trust-based interactions are also predicated on an ability to create and adapt models of others and their intentions. Such theory-of-mind processes engage a network of brain regions including the temporoparietal junction (TPJ), posterior cingulate cortex (PCC), and medial prefrontal cortex (mPFC). Together, these regions comprise the "social brain," which shares substantial overlap with the default-mode network (DMN; (Mars et al., 2012)). The DMN shows enhanced reactivity to social relative to non-social outcomes (Fareri et al., 2020) and may serve to prime us for engagement with the social world (Meyer, 2019). One possibility is that functional alterations within the DMN or between the DMN and reward-related networks may underlie the increased positivity and focus on beneficial social engagement in older adults, in turn contributing to an increased risk for exploitation (Castle et al., 2012; Harlé \& Sanfey, 2012; Spreng, Shoemaker, et al., 2017). 
Here, we sought to systematically investigate age-related differences in the effects of social closeness on trust behavior and the neural representation of reciprocity. In a pre-registered study, we implemented a variant of a trust game task used in a previous study (Fareri et al., 2015): participants played with a computer, a stranger, and a close friend who accompanied them to the experiment. While older adults may prioritize interactions with close others to meet emotional goals (e.g., maintaining a relationship), there may also be benefits to interacting with less close individuals, as we may lose members of our inner social networks with age (reviewed in Uchino \& Rook, 2020). For example, having larger numbers of weaker social ties as we enter older adulthood is more strongly predictive of lower levels of depression and higher levels of positive affect 10-15 years later than the number of close relationships one has (Huxhold et al., 2020). Based on these pieces of evidence, we hypothesized lower rates of trusting friends relative to strangers in older (ages 63-80) relative to younger adults (ages 18-34). We additionally expected blunted striatal responses to reciprocity from friends relative to strangers in older adults. We also hypothesized that age-related differences in striatal responses to reciprocity would be tied to increased DMN-striatal connectivity, which in turn would mediate expected age-related differences in trust behavior; we expected this mediation to be moderated by self-reported social closeness.

\section{Methods}

\section{Participants}

Fifty participants (26 young adults, ages 18-34; 24 older adults, ages 63-80) were recruited to participate in this study. This sample size was pre-registered (https://aspredicted.org/MVZ ODI), determined a priori before data collection, and limited largely by available funding for data collection for this project. We acknowledge that the relatively small sample size limits our ability to draw strong inferences, especially in analyses relating brain activation and self-reports of social closeness. Young adult participants were recruited primarily through the Temple University Psychology Department participant pool and received course credit (along with a task bonus in gift cards) for participation. Older adult participants were recruited using a range of efforts-reaching out to local community and senior centers, newspaper advertisements, and local flyers-and were compensated with Amazon gift cards for their participation (\$25 per hour of participation for MRI participants, $\$ 15$ per hour for both their friends and recruited confederates to act as strangers; bonus payment for MRI participants and their friends varied across individuals based on randomly chosen outcomes paid out at the end of the experimental session). All participants were screened before data collection to rule out current major psychiatric or neurologic illness, as well as MRI contraindications. Older adults were screened to rule out dementia using the Telephone Interview for Cognitive Status, with a score under 30 meeting exclusion criteria (Brandt and Folstein, 2012). All participants included in analyses had at least 3 usable runs of the task. These exclusions left a final sample of 48 total participants, with 26 younger adults (mean age: 23.2 years, SD: 4.07 years; $35 \%$ male) and 22 older adults (mean age: 69.3 years; SD: 4.38 
years; $50 \%$ male). All participants gave written informed consent as part of a protocol approved by the Institutional Review Board of Temple University.

\section{Procedures}

Participants completed two appointments. During the first appointment, participants underwent a mock MRI scan to help control for motion and acclimate to the scanner. They also completed a brief neuropsychological test battery including measures of estimated premorbid intelligence, specific cognitive domains (e.g., attention, executive functioning, episodic memory, language), and self-reported everyday functioning (older adults only). Participants completed a second appointment with a self-selected friend of the same identified sex and age group (within 5-10 years, who was not a family member or spouse). During the second appointment, participants completed a 45-minute survey that included measures of mood, media usage, and emotion regulation (not presented here) as well as questions about perceived social closeness with their friend and the stranger (Inclusion of Other in Self Scale (IOS); Aron et al., 1992)), and basic demographics.

\section{Experimental Paradigm}

After completing the survey, participants and their friends were introduced to a sex- and agematched (within 10 years) stranger (confederate) and were then trained on the Trust Game task (adapted from (Fareri et al. 2015)). MRI participants were told that they would be playing a game called the investment game in real time with their friend, the stranger and a computer partner. On a given trial of the task, the MRI participant would play with one of their three partners as indicated by a photo and name presented on the screen. Participants were instructed that they would start each trial with $\$ 8$ and that they would have a choice between sending (investing) different proportions of that $\$ 8$ to their partner on a given trial. The amounts that could be sent varied on a trial to trial basis, ranging from $\$ 0-\$ 8$. Participants would have up to 3 seconds to indicate via a button press on an MRI compatible response box which of the two investment options they preferred. Participants were instructed that whatever amount they chose to invest would be multiplied by a factor of 3 (i.e., an investment of $\$ 6$ would become $\$ 18$ for the partner), and that their partner could decide to split the multiplied amount evenly with them (reciprocate) or keep it all for themselves (defect). Upon entering their response, participants would see a screen that said 'waiting', (1.5s) during which time they believed that their decision was being presented to their partner in another room in the research suite. After the waiting screen, a variable ISI was presented (mean $=1.42 \mathrm{~s}$ ), and participants were then notified (2s) whether their partner decided to split that amount evenly with them (reciprocate) or keep (defect) all of the money. Unbeknownst to participants, all outcomes were predetermined, and all partners were preprogrammed to reciprocate $50 \%$ of the time as per our previous work (Fareri et al., 2015).

After task training, the MRI participant, the stranger and close friend were split up into different rooms before beginning the actual experimental session. Participants underwent a 90-minute MRI scan that included up to 5 runs of the trust game task, as well as two additional, but 
separate tasks (not discussed here). Each run of the task consisted of 36 trials, with 12 trials per partner. See Figure $1 \mathrm{~A}$ and our previous work (Fareri et al., 2015) for task details.
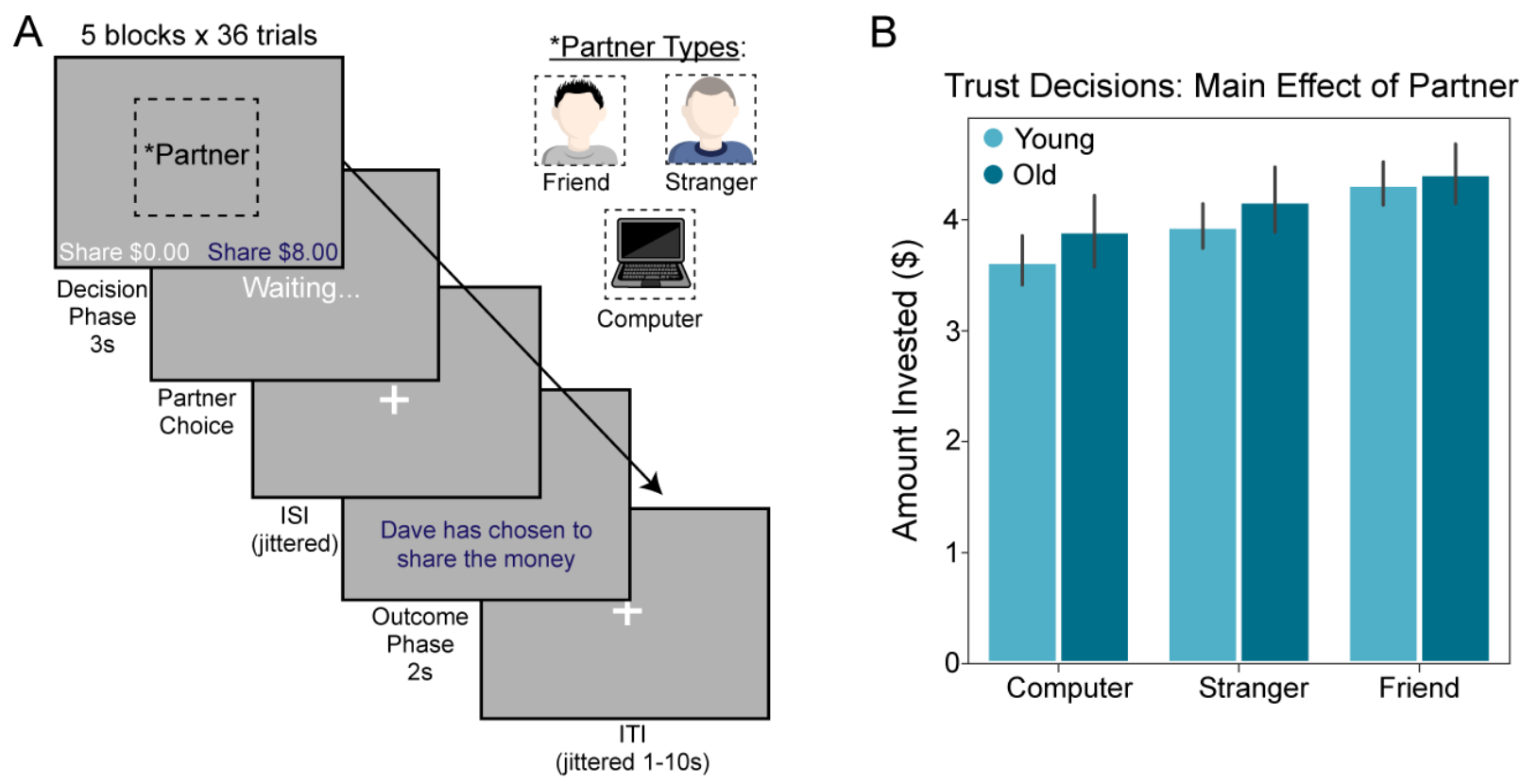

\section{Figure 1: Task and Behavior}

(A) We used an economic trust game task to measure trust. Participants played the role of the investor with three distinct investees of varying social closeness (friend, stranger, and computer as a nonsocial control). On each trial of the trust task, participants were first shown an image of their partner with a neutral expression (note that images shown here are cartoons for illustrative purposes only since journal policies prohibit showing actual photographs) and were given a choice to invest a greater or lesser amount of money with their partner. Money invested into the partner triples. When money was invested into the investee, the participant was then shown a screen indicating that the investee is contemplating the investment ("Waiting..."). After a brief jittered ISI, the participant is shown the outcome of the investee's decision (i.e., reciprocate and share the money evenly, or defect and take all of the money). (B) We examined whether partner identity impacted the amount invested, which serves as a metric of trust. We found that both older and younger participants invested money as a function of partner.

\section{Behavioral Analyses}

To ensure that we effectively manipulated social closeness, we conducted a 2 (partner) $\times 2$ (age group) repeated measures ANOVA on participants' IOS ratings of their friends and strangers. We examined participants' decisions to trust (i.e., invest) as a function of partner and age by conducting a 3 (Partner) x 2 (Age Group) repeated measures ANOVA (see 'Deviations from Pre-Registration section in Supplementary Methods) We also conducted this analysis controlling for individual differences in reaction time by z-scoring participants' mean reaction time during the decision phase and including these values as a covariate in an ANCOVA. This was done to 
ensure any potential differences in trust decisions were not accounted for by differences in reaction time during decision-making. We similarly performed a 3 (Partner) x 2 (Age Group) repeated measures ANOVA solely on participants' reaction time during the decision phase. We also performed additional exploratory analyses assessing differences in choice behavior and reaction time as a function of whether participants experienced reciprocity or a violation of trust on the previous trial.

\section{Neuroimaging Data Acquisition}

Neuroimaging data were collected at the Temple University Brain Research and Imaging Center (TUBRIC) using a 3.0 Tesla Siemens Prisma scanner equipped with a 20-channel phased array head coil. Functional images sensitive to blood-oxygenation-level-dependent (BOLD) contrast were acquired using a single-shot T2*-weighted echo-planar imaging sequence with slices roughly parallel to the axial plane collected in descending order [repetition time (TR): $2.02 \mathrm{~s}$; echo time (TE): 23 ms; matrix 74 x 74; voxel size: $2.97 \times 2.97 \times 2.80 \mathrm{~mm}$; 36 slices (15\% gap); flip angle: $76^{\circ}$. To facilitate co-registration and normalization of functional data, we also collected high-resolution T1-weighted structural scans (TR: 2.4 s; TE: 2.2 ms; matrix 192 x 192; voxel size: $1.0 \mathrm{~mm}^{3}$; 192 slices; flip angle: $8^{\circ}$ ) and $\mathrm{B}_{0}$ field maps (TR: $645 \mathrm{~ms}$; $\mathrm{TE}_{1}$ : $4.92 \mathrm{~ms}$; $\mathrm{TE}_{2}$ : $7.38 \mathrm{~ms}$; matrix $74 \times 74$; voxel size: $2.97 \times 2.97 \times 2.80 \mathrm{~mm}$; 36 slices, with $15 \%$ gap; flip angle: $60^{\circ}$ ). In addition, we also collected T2-weighted structural images (TR: $3.2 \mathrm{~s} ;$ TE: $567 \mathrm{~ms}$; matrix 192 x 192; voxel size: $1.0 \mathrm{~mm}^{3}$; 192 slices; flip angle: $120^{\circ}$ ); these images are included with our data on OpenNeuro.org, but we did not use them in our preprocessing or analyses.

\section{Preprocessing of Neuroimaging Data}

Neuroimaging data were converted to the Brain Imaging Data Structure (BIDS) using HeuDiConv version 0.5.4 (Halchenko et al., 2019). Results included in this manuscript come from preprocessing performed using fMRIPrep 20.1.0 (Esteban, Blair, et al., 2018; Esteban, Markiewicz, et al., 2018), which is based on Nipype 1.4.2 (K. Gorgolewski et al., 2011; K. J. Gorgolewski et al., 2018). The details described below are adapted from the fMRIPrep preprocessing details; extraneous details were omitted for clarity.

\section{Anatomical data preprocessing}

The T1-weighted image was corrected for intensity non-uniformity (INU) with N4BiasFieldCorrection (Tustison et al., 2010), distributed with ANTs 2.2.0 (Avants et al., 2008), and used as T1w-reference throughout the workflow. The T1w-reference was then skull-stripped with a Nipype implementation of the antsBrainExtraction.sh workflow (from ANTs), using OASIS30ANTs as target template. Brain tissue segmentation of cerebrospinal fluid (CSF), white-matter (WM) and gray-matter (GM) was performed on the brain-extracted T1w using FAST (FSL 5.0.9, (Zhang et al., 2001)). Volume-based spatial normalization to MNI152NLin2009cAsym standard space was performed through nonlinear registration with 
antsRegistration (ANTs 2.2.0), using brain-extracted versions of both T1w-reference and the T1w template. To this end, the ICBM 152 Nonlinear Asymmetrical template version 2009C (Fonov et al., 2009) template was selected for spatial normalization.

\section{Functional data preprocessing}

For each of the BOLD runs contained per subject, the following preprocessing steps were performed. First, a reference volume and its skull-stripped version were generated using a custom methodology of fMRIPrep (Esteban, Markiewicz, et al., 2018). Head-motion parameters with respect to the BOLD reference (transformation matrices, and six corresponding rotation and translation parameters) are estimated before any spatiotemporal filtering using mcflirt (FSL 5.0.9, (Jenkinson et al., 2002)). BOLD runs were slice-time corrected using 3dTshift from AFNI 20160207 (Cox \& Hyde, 1997). A B0-nonuniformity map (or fieldmap) was estimated based on a phase-difference map calculated with a dual-echo GRE (gradient-recall echo) sequence, processed with a custom workflow of SDCFlows inspired by the epidewarp.fsI script and further improvements in HCP Pipelines (Glasser et al., 2013). The fieldmap was then co-registered to the target EPI (echo-planar imaging) reference run and converted to a displacements field map (amenable to registration tools such as ANTs) with FSL's fugue and other SDCflows tools. Based on the estimated susceptibility distortion, a corrected EPI (echo-planar imaging) reference was calculated for a more accurate co-registration with the anatomical reference. The BOLD reference was then co-registered to the T1w-reference using FLIRT (FSL 5.0.9, (Jenkinson \& Smith, 2001)) with the boundary-based registration (Greve \& Fischl, 2009) costfunction. Co-registration was configured with nine degrees of freedom to account for distortions remaining in the BOLD reference. The BOLD time-series (including slice-timing correction when applied) were resampled onto their original, native space by applying a single, composite transform to correct for head-motion and susceptibility distortions. These resampled BOLD timeseries will be referred to as preprocessed BOLD in original space, or just preprocessed BOLD. The BOLD time-series were resampled into standard space, generating a preprocessed BOLD run in MNI152NLin2009cAsym space.

Additionally, a set of physiological regressors were extracted to allow for component-based noise correction (CompCor, (Behzadi et al., 2007)). Principal components are estimated after high-pass filtering the preprocessed BOLD time-series (using a discrete cosine filter with 128s cut-off) for the two CompCor variants: temporal (tCompCor) and anatomical (aCompCor). tCompCor components are then calculated from the top 5\% variable voxels within a mask covering the subcortical regions. This subcortical mask is obtained by heavily eroding the brain mask, which ensures it does not include cortical GM regions. For aCompCor, components are calculated within the intersection of the aforementioned mask and the union of CSF and WM masks calculated in T1w space, after their projection to the native space of each functional run (using the inverse BOLD-to-T1w transformation). Components are also calculated separately within the WM and CSF masks. For each CompCor decomposition, the k components with the largest singular values are retained, such that the retained components' time series are sufficient to explain 50 percent of variance across the nuisance mask (CSF, WM, combined, or temporal). The remaining components are dropped from consideration. As an additional 
confound, we also estimated framewise displacement (FD). FD was computed using the relative root mean square displacement between affines (Jenkinson et al., 2002).

All resamplings can be performed with a single interpolation step by composing all the pertinent transformations (i.e., head-motion transform matrices, susceptibility distortion correction when available, and co-registrations to anatomical and output spaces). Gridded (volumetric) resamplings were performed using antsApplyTransforms (ANTs), configured with Lanczos interpolation to minimize the smoothing effects of other kernels (Lanczos, 1964).

\section{Neuroimaging Analyses}

Neuroimaging analyses used FSL version 6.03 (Jenkinson et al., 2012; S. M. Smith et al., 2004). We specifically focused on two types of models (activation and connectivity) to quantify how reciprocated trust and social closeness were associated with BOLD responses. Both types of models were based on a general linear model with local autocorrelation (Woolrich et al., 2001). Our first model focused on the brain activation evoked during the trust task and used a total of nine regressors of interest. We used three regressors to model the decision phase (duration = response time) associated with each of the partners (computer, stranger, friend). To model brain activation associated with outcomes (reciprocate and defect) by partner, we used six additional regressors (duration $=1$ second). Each task-related regressor was convolved with the canonical hemodynamic response function. We also conducted offline analyses examining the relation between reward-related BOLD responses and self-reported social closeness (see Deviations from Pre-registration section, Supplementary Methods).

Our second type of model focused on the task-dependent connectivity associated with the trust task. To estimate these changes in connectivity, we used psychophysiological interaction (PPI) analysis (Friston et al., 1997; O'Reilly et al., 2012). Notably, recent meta-analytic work has shown that PPI reveals consistent and specific patterns of connectivity across multiple seed regions and psychological contexts (D. V. Smith et al., 2016; D. V. Smith \& Delgado, 2017). We first estimated a network PPI model that focused on task-dependent changes in connectivity with the DMN (Fareri et al., 2020; Utevsky et al., 2017). The DMN and nine additional networks, including the executive control network (ECN) were based on prior work (S. M. Smith et al., 2009). Network time courses were extracted with a spatial regression component of the dual regression approach (Filippini et al., 2009; Nickerson et al., 2017) and entered into a model with the nine task regressors from the activation model described above. PPI regressors were formed by multiplying each of the nine task regressors by the DMN regressor, yielding a total of 28 regressors.

We also conducted exploratory seed-based analyses using regions defined from our analyses that extant research implicates in reward and social processes, including the ventral striatum, vmPFC, FFA, TPJ and PCC (see Results). For each participant in these analyses, the average time course of a seed was extracted and entered as a regressor into a model with the nine task regressors from the activation model described above. PPI regressors were formed by 
multiplying each of the nine task regressors by the seed regressor, yielding a total of 19 regressors in each seed-based PPI model.

Both activation and connectivity models included a common set of confound regressors. We first modeled out missed responses by including an additional task-related regressor to account for the full duration of the choice screen. We also included additional regressors for the six motion parameters (rotations and translations), the first six aCompCor components explaining the most variance, non-steady state volumes, and the framewise displacement (FD) across time. Finally, high-pass filtering (128s cut-off) was achieved using a set of discrete cosine basis functions.

We combined data across runs, for each participant, using a fixed-effects model. Group-level analysis was carried out using FLAME (FMRIB's Local Analysis of Mixed Effects) Stage 1 and Stage 2 (Beckmann et al., 2003; Woolrich et al., 2004). Our group-level model focused on comparisons between older and younger groups; these comparisons included covariates to account for gender, temporal signal to noise ratio (tSNR), mean framewise displacement, and mean response time. All z-statistic images were thresholded and corrected for multiple comparisons using an initial cluster-forming threshold of $z \square>\square 3.1$ followed by a whole-brain corrected cluster-extent threshold of $\mathrm{p} \square<\square 0.05$, as determined by Gaussian Random Field Theory (Worsley, 2001).

\section{Results}

\section{Social closeness shapes trust decisions}

As a manipulation check, we investigated whether participants exhibited differences in selfreported social closeness to friends and strangers. A 2x2 repeated-measures ANOVA on IOS scores revealed a significant main effect of partner $\left(F_{(1,41)}=70.40, p<.001\right)$, such that participants reported feeling closer to friends $(M=4.50, S D=1.70)$ relative to strangers $(M=$ $2.14, S D=1.52$ ). Contrary to our hypotheses, we observed neither a significant interaction of partner and age group $\left(F_{(1,41)}=0.10, p=0.76\right)$, nor a main effect of age group $\left(F_{(1,41)}=2.53, p=\right.$ $.12)$, though the group difference is trending in the hypothesized direction of blunted effects in older adults $\left(\mathrm{M}_{\text {old }}=4.09, \mathrm{SD}=1.82, \mathrm{M}_{\text {young }}=4.85, \mathrm{SD}=1.54\right)$. We note that due to technical error, a number of participants $(n=5)$ lacked closeness ratings for the stranger and were unable to be included in this analysis.

Our first goal was to examine differences in trust behavior as a function of age and partner. To test pre-registered hypothesis 1.1, we conducted a 3 (partner) x 2 (age group) repeated measures ANOVA on the average amount of money invested during the trust game. Controlling for individual differences in overall reaction time, we observed partial support for pre-registered hypothesis 1.1: a significant main effect of partner on amount of money invested emerged $\left(F_{(2,90)}\right.$ $=18.49, p<.001$; see Figure 1B), such that participants sent more money to their friends relative to both strangers $\left(t_{(88)}=2.86, p<.01\right)$ and computers $\left(t_{(88)}=5.14, p<.001\right)$. This result replicates prior work from our group (Fareri et al., 2015). However, inconsistent with hypothesis 
1.1, we did not observe an interaction between partner and age $\left(F_{(2,90)}=2.16, p=0.12\right)$, though we did note a trending difference between older and younger adults in amount of money invested overall $\left(F_{(1,45)}=2.77, p=.10\right)$, with older adults investing marginally more money in their partners than younger adults. An additional exploratory ANOVA on choice behavior following reciprocity and violations of trust revealed that participants overall invested more with friends relative to other partners regardless of previous outcome $\left(F_{(2,92)}=7.38, p<.001\right)$ and invested more after reciprocity relative to violations of trust $\left(F_{(1,46)}=10.74, p<.002\right)$. We note that no significant interactions emerged between either factor and age, though we do note that a three-way interaction between partner, choice and age was approaching a trend $\left(F_{(2,92)}=1.89\right.$, $p=.156)$.

We also conducted a 3 (partner) x 2 (age group) repeated measures ANOVA on reaction time. This analysis revealed a significant effect of age $\left(F_{(1,46)}=7.60, p<.01\right)$, with older adults exhibiting significantly longer reaction times when making their decisions. A trending main effect of partner also emerged $\left(F_{(2,92)}=2.75, p=.069\right)$, such that participants responded more quickly when interacting with their friends relative to the computer $\left(t_{(92)}=2.34, p=.064\right)$. An additional exploratory ANOVA on reaction time as a function of outcomes on previous trials revealed that participants responded more quickly on trials with friends relative to other partners $\left(F_{(2,92)}=3.08\right.$, $p=.051)$ and more quickly after experiencing reciprocity $\left(F_{(1,46)}=15.58, p<.001\right)$. We interestingly observed a significant three-way interaction between partner, choice and age on reaction time $\left(F_{(2,92)}=3.16, p<.05\right)$, whereby younger adults show similarly quick reaction times after either reciprocity or violations of trust with all partners, whereas older adults show longer reaction times after experiencing violations of trust from computer partners, but similar reaction times regardless of previous outcome with social partners.

\section{fMRI Results: Trust Game Outcomes}

\section{Blunted striatal responses to social closeness in older adults}

Based on our expected behavioral results and our prior work, we were also interested in characterizing differences in task-based neural activation and connectivity specifically during experienced outcomes (i.e., reciprocity vs. defection of trust) in the trust game. We first hypothesized that participants would exhibit enhanced reward-related responses within the striatum when experiencing reciprocity from a close friend relative to other partners, and that this effect would be stronger in younger adults and associated with relationship closeness (pre-registered hypothesis 2.1). To test this hypothesis, we conducted a confirmatory whole-brain contrast of reciprocate $>$ defect. This analysis revealed robust activation of the bilateral ventral striatum (Rt. ventral striatum: $x, y, z=7.5,13.2$, -4.4; Lt. ventral striatum: $x, y, z=-10.3,16.2,-1.2$; see Fig. 2A), among other regions, including vmPFC, mPFC, PCC and occipital cortex (see Table 1 and https://identifiers.org/neurovault.image:512088 for more information). 

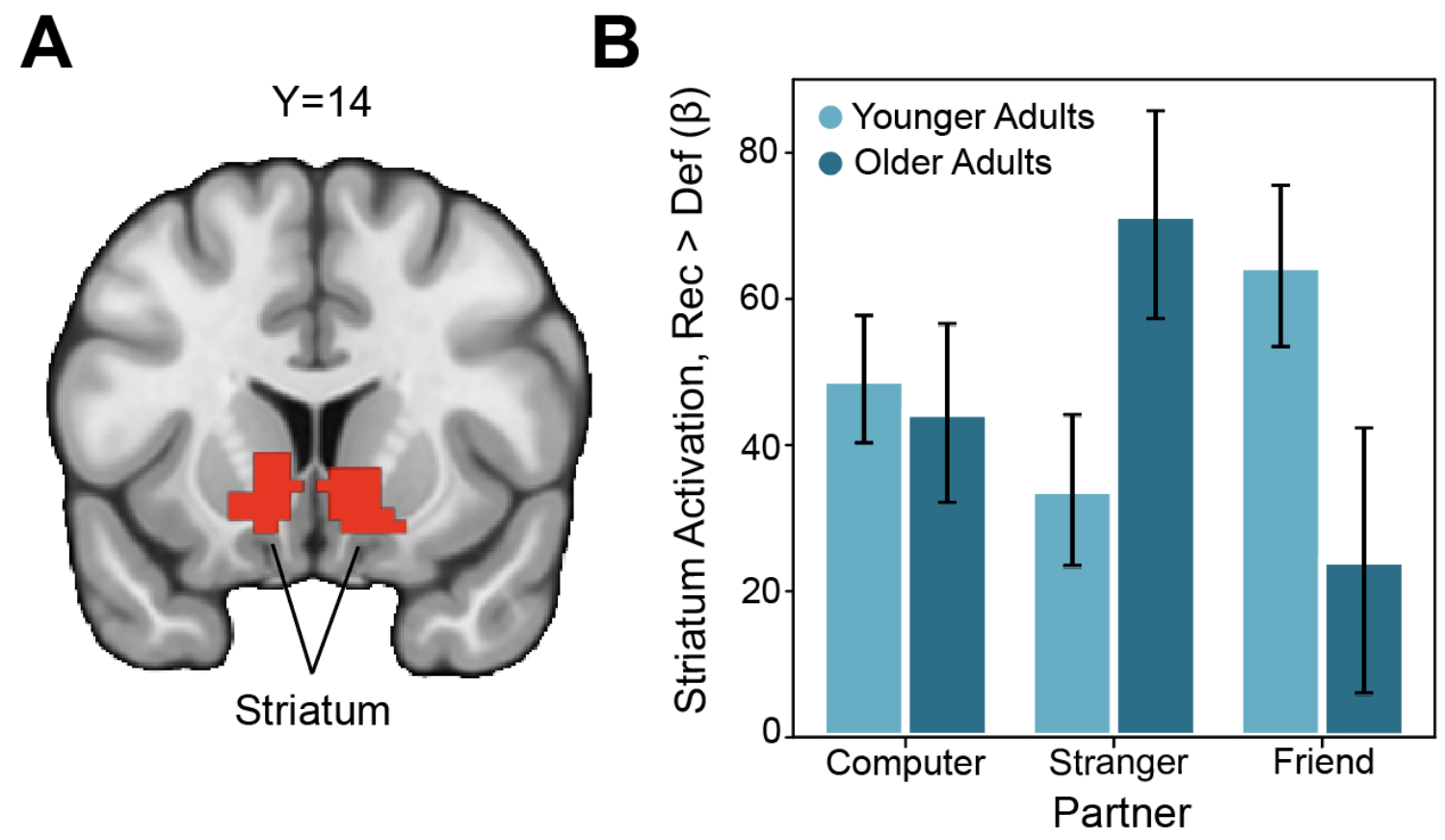

\section{Figure 2: Blunted Effects of Social Closeness on Striatal Social Reward Responses in Older Adults.}

(A) We identified voxels in the striatum whose activation increased for reciprocate outcomes relative to defect outcomes, irrespective of age. The overlaying $Z$ statistic image was thresholded parametrically (Gaussian Random Field Theory) using GRF-theory-based maximum height thresholding (i.e., voxel level threshold) with a (corrected) significance threshold of $P=0.05$ (Thresholded:

https://identifiers.org/neurovault.image:512088; Unthresholded:

https://identifiers.org/neurovault.image:512063). (B) We interrogated this striatal region further to probe for group differences in the effects of partner on reward processing. Although younger adults exhibited an enhanced striatal response to the contrast of reciprocate $>$ defect when the partner was a friend relative to a stranger, older adults exhibited the opposite effect, suggesting that the effects of social closeness on reward related neural responses are blunted in older adults.

We next conducted a 2 (age group) x 3 (partner) repeated-measures ANOVA on extracted parameter estimates from the bilateral ventral striatum to investigate age differences in processing of trust game outcomes. We observed a significant partner $\mathrm{x}$ group interaction $\left(F_{(2,92)}\right.$ $=5.94, p=.004$; see Figure $2 \mathrm{~B}$ ): older adults exhibited diminished striatal activation when experiencing reciprocate relative to defect outcomes from friends as compared to strangers $\left(t_{(46)}\right.$ $=-2.68, p=.01)$, whereas young adults demonstrated a trend toward the opposite pattern $\left(t_{(46)}\right.$ $=1.89, p=.065$ ), consistent with patterns demonstrated in prior work from our group (Fareri et al., 2015). We note that re-running these analyses including tSNR, mean framewise displacement (FD) and sex as covariates reduced the partner $x$ group interaction effect to a trend $(F(2,92)=2.30, p=.107)$.

Last, we examined whether differences in self-reported social closeness with friends and strangers was associated with differences in the striatal response to trust game outcomes with 
friends and strangers. A linear regression regressing the difference in striatal BOLD for reciprocate $>$ defect for friends $>$ strangers on the difference in reported social closeness with those same partners revealed a trend of an effect $(b=13.00, \mathrm{SE}=7.86, t=1.66, p=.104)$ : the closer participants felt to their friends relative to strangers, the greater the difference in striatal BOLD responses to reciprocity $>$ defect outcomes experienced with friends relative to strangers. We note that this is a weak but encouraging effect, and that including tSNR, FD and sex as covariates diminishes the strength of this effect $(p=.18)$. Taken together these results provide partial support for pre-registered hypothesis 2.1 .

\section{Altered cortical responses to social closeness in older adults}

We also conducted an additional exploratory whole-brain interaction contrast to highlight regions demonstrating age-related differences in responses to trust game outcomes. This analysis revealed significant clusters of activation in the anterior insula, posterior cingulate and both dorsal and ventral medial prefrontal cortex (see Table 1). Extracting parameter estimates from the insula $(x, y, z=37,25,8)$ revealed a reduced response to defect relative to reciprocate outcomes specifically in the stranger condition in older adults (Fig 3A, B). Older adults also demonstrated a reduced response to reciprocate relative to defect outcomes experienced with friends in the PCC (x, y, z = 4, -58, 28; Fig 3C, D). 


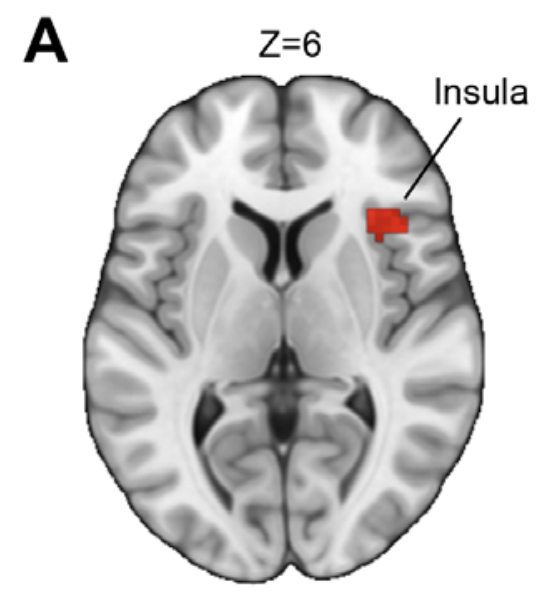

B
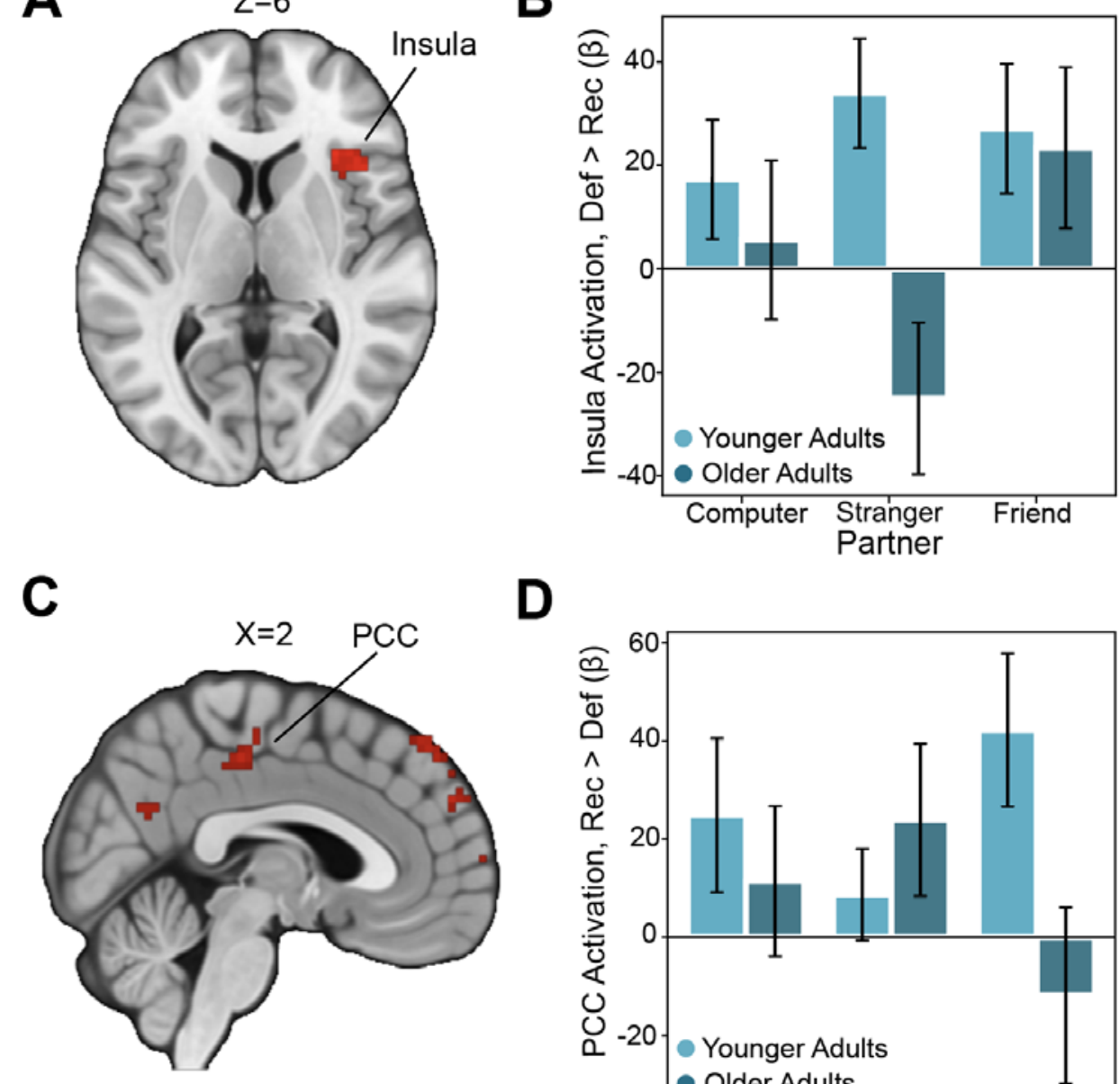

D

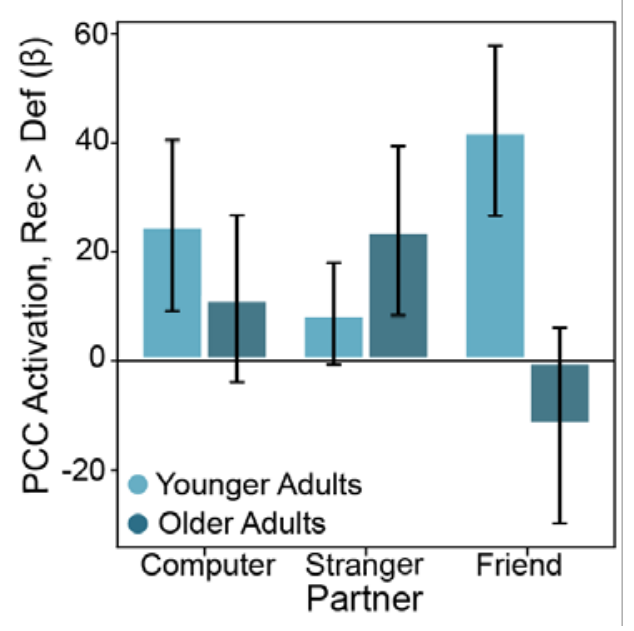

Figure 3: Age-related Differences in Neural Responses to Social Outcomes

(A) We found that the anterior insula exhibited a larger response to defect outcomes relative to reciprocate outcomes in younger adults (plotted here as defect > reciprocate), consistent with the idea that older adults are less sensitive to outcomes associated with losses (Thresholded:

https://identifiers.org/neurovault.image:512048; Unthresholded:

https://identifiers.org/neurovault.image:512076). (B) For descriptive purposes, we extracted beta values within this anterior insula region and confirmed that older adults showed a blunted response to defect outcomes. (C) In the opposite contrast (reciprocate > defect), we found several regions whose outcomerelated activation was enhanced in younger adults (Thresholded: https://identifiers.org/neurovault.image:512037; Unthresholded:

https://identifiers.org/neurovault.image:512065). (D) For descriptive purposes, we extracted the response within the posterior cingulate cortex (PCC) and confirmed that the response to reciprocation in this region was enhanced in younger adults. We note that $Z$ statistic images were thresholded parametrically (Gaussian Random Field Theory) using clusters determined by Z>3.1 and a (corrected) cluster significance threshold of $P=0.05$. 


\section{Older adults exhibit enhanced network connectivity during trust game outcomes with friends}

Our task-based activation analyses revealed engagement of both reward related circuits and regions comprising the default mode network (e.g., PCC, mPFC) during experiences of reciprocity relative to violations of trust. As outlined in our pre-registration, we predicted that we would observe age-related differences in default mode connectivity, specifically with the striatum, when experiencing reciprocity relative to defection from different partners $(\mathrm{H} 2.2)$. To test this prediction, we conducted a generalized network psychophysiological interaction (nPPI) analysis during the outcome phase of the task using the DMN as our seed network (cf. Fareri et al., 2020). A contrast of reciprocate $>$ defect as a function of social closeness (friend $>$ computer) and age (young > old) revealed enhanced connectivity between the DMN and TPJ ( $x$, $y, z=64,-40,24.5$; see Fig. 4) when experiencing reciprocity from friends relative to the computer in older, compared to younger adults, suggesting that TPJ is more tightly integrated within the DMN in this particular task context. This analysis also revealed enhanced DMN coupling with the supplementary motor area and the occipital pole (see Table 1 and https://identifiers.org/neurovault.image:512032). Interestingly, we did not observe enhanced connectivity between the DMN and striatum as predicted in pre-registered hypothesis 2.2 and as such we did not pursue our final pre-registered hypothesis $(\mathrm{H} 2.3)$, which posited that DMNstriatal connectivity would mediate partner related effects in trust behavior.
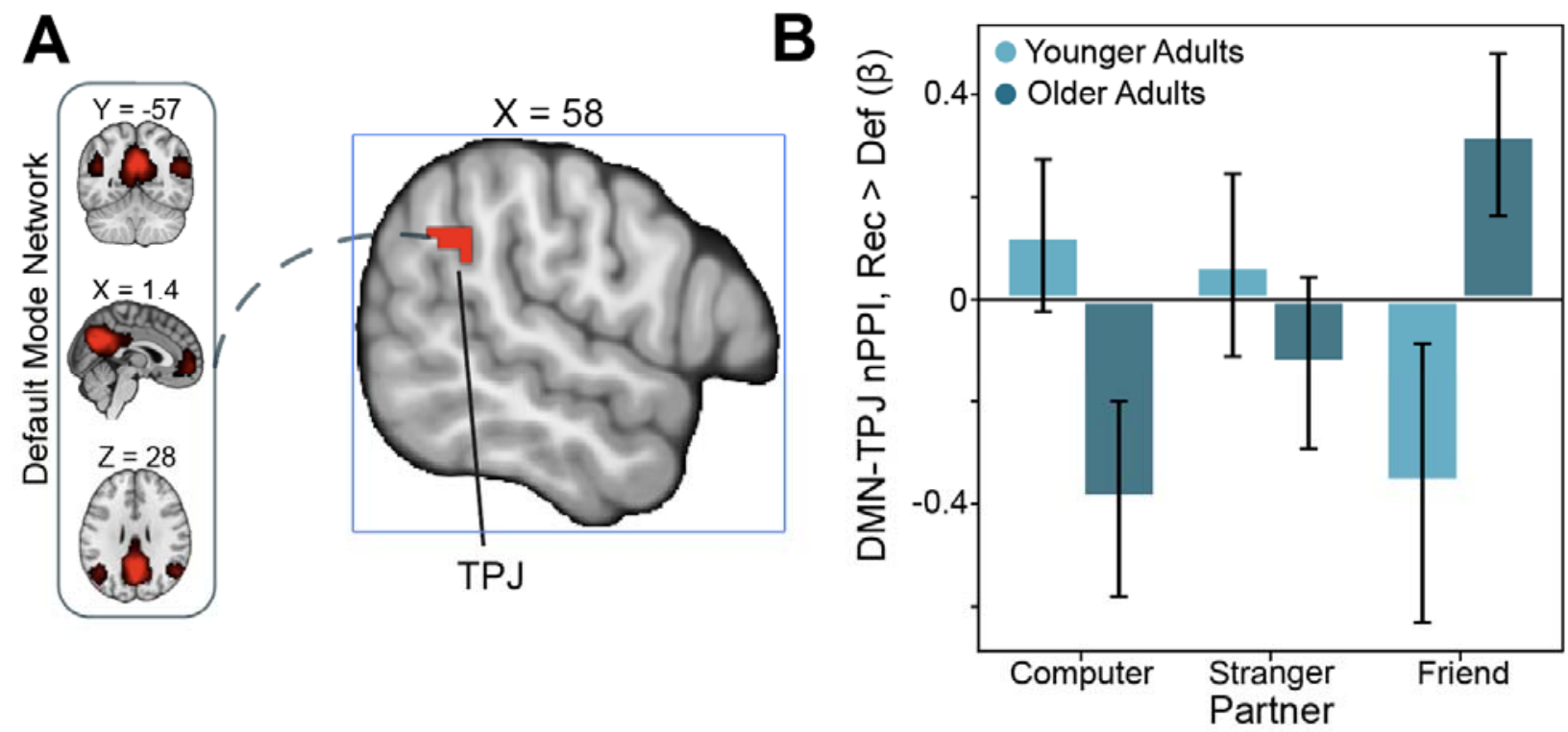

Figure 4: Older Adults Show Enhanced DMN-TPJ Connectivity as a Function of Social Closeness

(A) We used a network psychophysiological interaction (nPPl) analysis with the default mode network $(D M N)$ as a seed. We examined connectivity with DMN as a function of age differences and social closeness (friend $>$ computer) during the outcome phase (reciprocate $>$ defect). This double subtraction analysis [(friend reciprocate $>$ friend defect) $>$ (computer reciprocate $>$ computer defect)] indicated that DMN connectivity with the right temporal-parietal junction (TPJ) was enhanced in older adults (Thresholded: https://identifiers.org/neurovault.image:512032; Unthresholded: https://identifiers.org/neurovault.image:512035). (B) For illustrative purposes, we extracted the parameter 
estimates within this TPJ region. We note that $Z$ statistic images were thresholded parametrically (Gaussian Random Field Theory) using clusters determined by Z>3.1 and a (corrected) cluster significance threshold of $P=0.05$.

We also explored task-based connectivity of the Executive Control Network (ECN) during the processing of trust game outcomes. This exploratory analysis was motivated by previous work implicating the ECN in reward-processing and goal directed behavior more generally (Fareri et al., 2020; Grill et al., 2021; Waltz et al., 2013). We conducted a network PPI contrast of reciprocate $>$ defect for friends $>$ strangers as a function of age group. Here, we found that relative to younger adults, older adults demonstrated increased connectivity between the ECN and a region encompassing mid/posterior insula ( $x, y, z=34,4$, -4; see Table 1) when experiencing defect outcomes with one's friend, but not with the stranger or computer.

In a control analysis, we examined whether these network connectivity effects were due to the template networks being derived from a younger and independent sample (S. M. Smith et al., 2009). We therefore used independent component analyses to identify networks in our sample (see Supplementary Methods). Results from both control analyses (DMN-TPJ, ECNinsula) partially replicated the patterns observed in our whole brain results (see Supplementary Results).

\section{Young adults demonstrate enhanced vmPFC-hippocampus connectivity during reciprocity}

Finally, we also conducted additional exploratory seed-based PPI analyses investigating group differences in responses to trust game outcomes as a function of social closeness using the following seeds: FFA, vmPFC and ventral striatum, TPJ and PCC. Robust group differences emerged (see the NeuroVault associated with this study

(https://identifiers.org/neurovault.collection:10447) for relevant maps), but here we highlight one result of interest from an analysis with the vmPFC as a seed region. This analysis interestingly revealed increased vmPFC-hippocampus (HPC; Right HPC: $x, y, z=22,-14$, -20.5; Left HPC: $28,-8,-24)$ connectivity in younger relative to older adults during reciprocate relative to defect outcomes (see Figure 5 and Table 1).
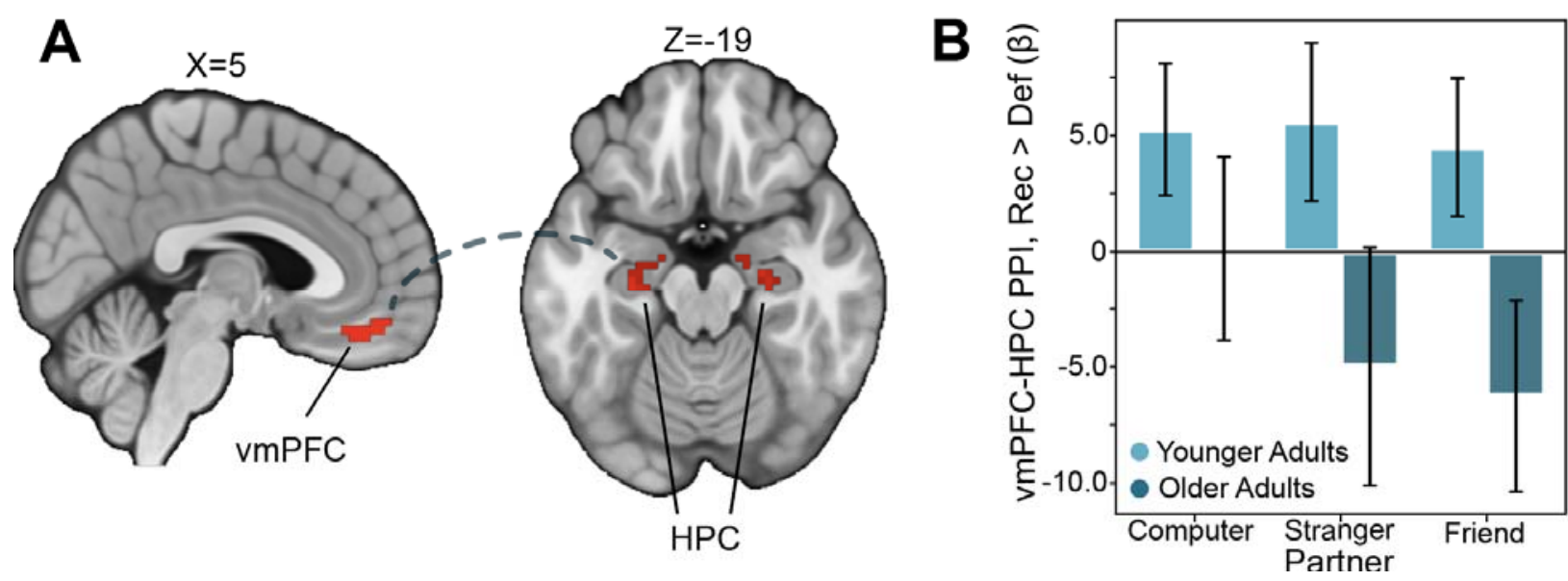


\section{Figure 5: Reduced vmPFC-Hippocampal Connectivity in Older Adults}

(A) In an exploratory seed-based PPI analysis, we examined age-related differences in connectivity with vmPFC. We found that older adults exhibited reduced vmPFC connectivity with a hippocampal (HPC) cluster extending into the amygdala during reciprocate relative to defect outcomes (Thresholded: https://identifiers.org/neurovault.image:512046; Unthresholded: https://identifiers.org/neurovault.image:512074). (B) Interrogation of this hippocampal cluster revealed that the pattern was stable across all partner types. We note that $Z$ statistic images were thresholded parametrically (Gaussian Random Field Theory) using clusters determined by Z>3.1 and a (corrected) cluster significance threshold of $P=0.05$.

\section{Discussion}

Older adulthood is often associated with a heightened focus on experiences with close others in conjunction with shrinking social networks, particularly when emotional goals are prioritized (Carstensen, 1992; Isaacowitz et al., 2021). This changing social world thus colors many daily decisions, including trusting a range of individuals with assets, potentially creating opportunities for exploitation and abuse (Spreng et al., 2016). Although decision neuroscience has made significant progress in characterizing how age-related differences in affect and motivation contribute to decision making (Samanez-Larkin \& Knutson, 2015), relatively less is known about age-related differences in social decision-making and social outcome processing. Using an iterated trust game in which participants played with three partners who varied in social closeness with the participant (computer, stranger, friend), we show that while overall, participants invested more with close others, consistent with prior work (Fareri et al., 2015; Webb et al., 2016), younger and older adults demonstrated similar rates of trust with friends and strangers. All participants were quicker to decide when interacting with friends relative to other partners, and more so after experiencing reciprocity, though older adults in general were slower to make decisions overall. Interestingly, neural processing of reciprocity and defection varied with respect to age and social closeness. Striatal responses to reciprocated trust from friends relative to strangers and computers were blunted in older adults relative to younger adults, and older adults also showed enhanced DMN connectivity with the rTPJ during reciprocated trust from friends compared to computers. Taken together, these results suggest that older adults demonstrate altered representations of social closeness within financial exchanges involving trust, which may have downstream effects for their ability to adapt their behavior in the longterm.

Altered recruitment of corticostriatal circuits in older adults when processing reciprocity and defection is partially consistent with earlier work looking at social outcome processing in aging. Early investigations noted that relative to younger adults, older adults demonstrated altered recruitment of corticostriatal circuits (e.g., dorsolateral PFC, insula) when faced with unfair offers in an ultimatum game relative to younger adults (Harlé \& Sanfey, 2012), and an enhanced anticipatory response in the ventral striatum to social rewards in older relative to younger adults (Rademacher et al., 2014). Findings have also shown reduced striatal activation in older adults when exposed to social outcomes that are inconsistent with initial impressions of others (Suzuki 
et al., 2019). Here, differences in social value-related signaling in the ventral striatum and the insula in older adults may suggest an inappropriate weighting of social outcomes with different social partners that may have implications for the increased susceptibility to financial exploitation in this population.

Our study also importantly builds on evidence (Hughes et al., 2020; Spreng, Cassidy, et al., 2017; Weissberger et al., 2020) suggesting that older adults at heightened risk for financial exploitation show altered activation and connectivity in regions supporting social cognition. For example, decreased mPFC activation and weaker DMN connectivity has been related to diminished mentalizing about unknown others in older relative to younger adults (Cassidy et al., 2021; Hughes et al., 2019). Here, we report enhanced DMN-TPJ connectivity in older adults when experiencing reciprocity from friends relative to strangers. Coupled with reported stronger ingroup (vs. outgroup) trust bias in older adults (Cassidy et al., 2020), the tighter integration of TPJ with the DMN during experienced reciprocity with friends here may suggest an augmented ability to incorporate social outcomes into models of known close others. We also found reduced vmPFC-HPC connectivity in older adults during experiences of reciprocity, which, when considered in light of evidence of reduced hippocampal involvement in feedback-based learning and memory in older adults (Lighthall et al., 2018), may suggest significant changes in the ways in which social and value-related information during trust-based interactions are integrated to inform social learning in aging samples. Future work may examine how age-related connectivity changes during social outcome processing may relate to neural differences during trust-related decision-making as a function of social closeness.

We note that our results merit further consideration of a few important caveats. First, we observed minimal age-related differences in willingness to trust others as a function of social closeness. Though this could be partly attributed to our small sample size (we were powered to detect moderate to large effects), it is also possible that age-related differences in behavior are more subtle, potentially requiring advanced computational methods to estimate latent factors in the decision-making process (Miletić et al., 2020). An alternative possibility is that because the choices in this task were designed to assess levels of trust in one given individual at a time, there were benefits to placing trust in a stranger, so as to learn about them (Isaacowitz et al., 2021). While such an interpretation is at odds with some theories of social reorientation in older adulthood (Carstensen, 1992), it is in line with suggestions of the benefits of maintaining less close relationships in older adulthood (Huxhold et al., 2020). We also note that inconsistencies are often reported with respect to age-related differences in trust decisions-older adults have been observed to both invest more with trustees (Bailey et al., 2016), and to demonstrate no differences relative to younger adults in investment behavior (Bailey et al., 2015)—while other findings note that older adults may be less able to effectively adapt behavior after negative (i.e., betrayal of trust) social outcomes (Bailey et al., 2019; Frazier et al., 2021). These differences may be in part due to variability in experimental design. Future work may implement alternative two-stage designs (Daw et al., 2011) in which older adults have the option to choose to interact on a given trial with a close or unknown other before deciding how much to invest, which may help disentangle some of these inconsistencies. In addition, the effects of social closeness on behavior could be influenced by other aspects of the relationships that we did not assess, 
including social network size (Kwak et al., 2018) and relationship quality (Santini et al., 2015), or by other aspects of cognition (i.e., episodic memory; see Suzuki, 2018). Last, age-related differences in brain activation and connectivity could be linked to confounding variables which we did not assess. We attempted to control for this possibility by including covariates relating to gender, head motion, data quality, and response time. Nevertheless, it is possible that other variables, such as white matter integrity and vascular health (Prins \& Scheltens, 2015), may have contributed to the age-related neural differences we observed.

Despite these limitations, our results support the conclusion that older adults exhibit altered neural responses to social closeness during trust-based social interactions. We believe that efforts to characterize age-related differences in these types of decisions may have implications for vulnerability to social victimization and abuse among the elderly, particularly financial exploitation (Lichtenberg, 2016; Lichtenberg et al., 2013; Spreng et al., 2016). Although financial exploitation is a multifaceted issue that can occur in a wide range of scenarios (Beals et al., 2015; Nguyen et al., 2021), many instances of financial exploitation may stem from placing too much trust in others (Nguyen et al., 2021; Shao et al., 2019). We speculate that future efforts to understand how older adults modulate decision making and reward processing as a function of social closeness will lay the groundwork for identifying novel risk factors for financial exploitation. 


\section{References}

Andrews-Hanna, J. R., Snyder, A. Z., Vincent, J. L., Lustig, C., Head, D., Raichle, M. E., \& Buckner, R. L. (2007). Disruption of Large-Scale Brain Systems in Advanced Aging. Neuron, 56(5), 924-935. https://doi.org/10.1016/j.neuron.2007.10.038

Aron, A., Aron, E. N., \& Smollan, D. (1992). Inclusion of other in the self scale and the structure of interpersonal closeness. Journal of Personality and Social Psychology, 63(4), 596.

Avants, B. B., Epstein, C. L., Grossman, M., \& Gee, J. C. (2008). Symmetric diffeomorphic image registration with cross-correlation: Evaluating automated labeling of elderly and neurodegenerative brain. Medical Image Analysis, 12(1), 26-41. https://doi.org/10.1016/j.media.2007.06.004

Bailey, P. E., Petridis, K., McLennan, S. N., Ruffman, T., \& Rendell, P. G. (2019). Age-Related Preservation of Trust Following Minor Transgressions. The Journals of Gerontology: Series B, 74(1), 74-81. https://doi.org/10.1093/geronb/gbw141

Bailey, P. E., Slessor, G., Rieger, M., Rendell, P. G., Moustafa, A. A., \& Ruffman, T. (2015). Trust and trustworthiness in young and older adults. Psychology and Aging, 30(4), 977986. https://doi.org/10.1037/a0039736

Bailey, P. E., Szczap, P., McLennan, S. N., Slessor, G., Ruffman, T., \& Rendell, P. G. (2016). Age-related similarities and differences in first impressions of trustworthiness. Cognition and Emotion, 30(5), 1017-1026. https://doi.org/10.1080/02699931.2015.1039493

Beals, M., DeLiema, M., \& Deevy, M. (2015). Framework for a taxonomy of fraud. Financial Fraud Research Center.

Beckmann, C. F., Jenkinson, M., \& Smith, S. M. (2003). General multilevel linear modeling for group analysis in FMRI. Neurolmage, 20(2), 1052-1063. https://doi.org/10.1016/S1053$8119(03) 00435-X$

Behzadi, Y., Restom, K., Liau, J., \& Liu, T. T. (2007). A component based noise correction 
method (CompCor) for BOLD and perfusion based fMRI. Neurolmage, 37(1), 90-101. https://doi.org/10.1016/j.neuroimage.2007.04.042

Bellucci, G., Chernyak, S. V., Goodyear, K., Eickhoff, S. B., \& Krueger, F. (2016). Neural signatures of trust in reciprocity: A coordinate-based meta-analysis. Human Brain Mapping, 38(3), 1233-1248.

Burr, D. A., Castrellon, J. J., Zald, D. H., \& Samanez-Larkin, G. R. (2021). Emotion dynamics across adulthood in everyday life: Older adults are more emotionally stable and better at regulating desires. Emotion, 21(3), 453-464. https://doi.org/10.1037/emo0000734

Carstensen, L. (1992). Social and emotional patterns in adulthood: Support for socioemotional selectivity theory. Psychology and Aging, 7(3), 331-338. https://doi.org/10.1037/08827974.7.3.331

Carstensen, L. (1995). Evidence for a Life-Span Theory of Socioemotional Selectivity. Current Directions in Psychological Science, 4(5), 151-156. https://doi.org/10.1111/14678721.ep11512261

Cassidy, B. S., Hughes, C., \& Krendl, A. C. (2020). A stronger relationship between reward responsivity and trustworthiness evaluations emerges in healthy aging. Aging, Neuropsychology, and Cognition, 0(0), 1-18. https://doi.org/10.1080/13825585.2020.1809630

Cassidy, B. S., Hughes, C., \& Krendl, A. C. (2021). Age differences in neural activity related to mentalizing during person perception. Aging, Neuropsychology, and Cognition, 28(1), 143-160. https://doi.org/10.1080/13825585.2020.1718060

Castle, E., Eisenberger, N. I., Seeman, T. E., Moons, W. G., Boggero, I. A., Grinblatt, M. S., \& Taylor, S. E. (2012). Neural and behavioral bases of age differences in perceptions of trust. Proceedings of the National Academy of Sciences, 109(51), 20848-20852.

Chang, L. J., Doll, B. B., van t Wout, M., Frank, M. J., \& Sanfey, A. G. (2010). Seeing is believing: Trustworthiness as a dynamic belief. Cognitive Psychology, 61(2), 87-105. 
Charles, S. T. (2010). Strength and vulnerability integration: A model of emotional well-being across adulthood. Psychological Bulletin, 136(6), 1068-1091. https://doi.org/10.1037/a0021232

Chen, P.-H. A., Fareri, D., Guroglu, B., Delgado, M. R., \& Chang, L. J. (2021). Towards a Neurometric-based Construct Validity of Trust. BioRxiv, 2021.07.04.451074. https://doi.org/10.1101/2021.07.04.451074

Cox, R. W., \& Hyde, J. S. (1997). Software tools for analysis and visualization of fMRI data. NMR in Biomedicine, 10(4-5), 171-178. https://doi.org/10.1002/(SICI)10991492(199706/08)10:4/5<171::AID-NBM453>3.0.CO;2-L

Daw, N. D., Gershman, S. J., Seymour, B., Dayan, P., \& Dolan, R. J. (2011). Model-Based Influences on Humans' Choices and Striatal Prediction Errors. Neuron, 69(6), 12041215. https://doi.org/10.1016/j.neuron.2011.02.027

Devitt, A. L., \& Schacter, D. L. (2020). Looking on the Bright Side: Aging and the Impact of Emotional Future Simulation on Subsequent Memory. The Journals of Gerontology: Series B, 75(9), 1831-1840. https://doi.org/10.1093/geronb/gbz041

Esteban, O., Blair, R., Markiewicz, C. J., Berleant, S. L., Moodie, C., Ma, F., Isik, A. I., Erramuzpe, A., Kent, M., James D. andGoncalves, DuPre, E., Sitek, K. R., Gomez, D. E. P., Lurie, D. J., Ye, Z., Poldrack, R. A., \& Gorgolewski, K. J. (2018). FMRIPrep. Software. https://doi.org/10.5281/zenodo.852659

Esteban, O., Markiewicz, C., Blair, R. W., Moodie, C., Isik, A. I., Erramuzpe Aliaga, A., Kent, J., Goncalves, M., DuPre, E., Snyder, M., Oya, H., Ghosh, S., Wright, J., Durnez, J., Poldrack, R., \& Gorgolewski, K. J. (2018). fMRIPrep: A robust preprocessing pipeline for functional MRI. Nature Methods. https://doi.org/10.1038/s41592-018-0235-4

Fareri, D. S. (2019). Neurobehavioral Mechanisms Supporting Trust and Reciprocity. Frontiers in Human Neuroscience, 13. https://doi.org/10.3389/fnhum.2019.00271

Fareri, D. S., Chang, L. J., \& Delgado, M. R. (2012). Effects of direct social experience on trust 
decisions and neural reward circuitry. Frontiers in Neuroscience, 6, 148-17.

Fareri, D. S., Chang, L. J., \& Delgado, M. R. (2015). Computational Substrates of Social Value in Interpersonal Collaboration. Journal of Neuroscience, 35(21), 8170-8180. https://doi.org/10.1523/JNEUROSCl.4775-14.2015

Fareri, D. S., Smith, D. V., \& Delgado, M. R. (2020). The influence of relationship closeness on default-mode network connectivity during social interactions. Social Cognitive and Affective Neuroscience, 15(3), 261-271. https://doi.org/10.1093/scan/nsaa031

Filippini, N., Maclntosh, B. J., Hough, M. G., Goodwin, G. M., Frisoni, G. B., Smith, S. M., Matthews, P. M., Beckmann, C. F., \& Mackay, C. E. (2009). Distinct patterns of brain activity in young carriers of the APOE- $\varepsilon 4$ allele. Proceedings of the National Academy of Sciences, 106(17), 7209-7214.

Fonov, V., Evans, A., McKinstry, R., Almli, C., \& Collins, D. (2009). Unbiased nonlinear average age-appropriate brain templates from birth to adulthood. Neurolmage, 47, Supplement 1, S102. https://doi.org/10.1016/S1053-8119(09)70884-5

Fouragnan, E., Chierchia, G., Greiner, S., Neveu, R., Avesani, P., \& Coricelli, G. (2013). Reputational priors magnify striatal responses to violations of trust. Journal of Neuroscience, 33(8), 3602-3611.

Frazier, I., Lin, T., Liu, P., Skarsten, S., Feifel, D., \& Ebner, N. C. (2021). Age and intranasal oxytocin effects on trust-related decisions after breach of trust: Behavioral and brain evidence. Psychology and Aging, 36(1), 10. https://doi.org/10.1037/pag0000545

Friston, K. J., Buechel, C., Fink, G. R., Morris, J., Rolls, E., \& Dolan, R. J. (1997).

Psychophysiological and Modulatory Interactions in Neuroimaging. Neurolmage, 6(3), 218-229. https://doi.org/10.1006/nimg.1997.0291

Glasser, M. F., Sotiropoulos, S. N., Wilson, J. A., Coalson, T. S., Fischl, B., Andersson, J. L., Xu, J., Jbabdi, S., Webster, M., Polimeni, J. R., Van Essen, D. C., \& Jenkinson, M. (2013). The minimal preprocessing pipelines for the Human Connectome Project. 
Neurolmage, 80, 105-124. https://doi.org/10.1016/j.neuroimage.2013.04.127

Gorgolewski, K., Burns, C. D., Madison, C., Clark, D., Halchenko, Y. O., Waskom, M. L., \&

Ghosh, S. (2011). Nipype: A flexible, lightweight and extensible neuroimaging data processing framework in Python. Frontiers in Neuroinformatics, 5, 13.

https://doi.org/10.3389/fninf.2011.00013

Gorgolewski, K. J., Esteban, O., Markiewicz, C. J., Ziegler, E., Ellis, D. G., Notter, M. P., Jarecka, D., Johnson, H., Burns, C., Manhães-Savio, A., Hamalainen, C., Yvernault, B., Salo, T., Jordan, K., Goncalves, M., Waskom, M., Clark, D., Wong, J., Loney, F., ... Ghosh, S. (2018). Nipype. Software. https://doi.org/10.5281/zenodo.596855

Greve, D. N., \& Fischl, B. (2009). Accurate and robust brain image alignment using boundarybased registration. Neurolmage, 48(1), 63-72. https://doi.org/10.1016/j.neuroimage.2009.06.060

Grill, F., Nyberg, L., \& Rieckmann, A. (2021). Neural correlates of reward processing: Functional dissociation of two components within the ventral striatum. Brain and Behavior, 11(2), e01987. https://doi.org/10.1002/brb3.1987

Halchenko, Y., Goncalves, M., Castello, M. V. di O., Ghosh, S., Hanke, M., Dae, Amlien, I., Brett, M., Salo, T., Gorgolewski, C., pvelasco, Stadler, J., Kaczmarzyk, J., lee, john, Lurie, D., Pellman, J., Melo, B., Poldrack, B., Nielson, D., ... Feingold, F. (2019). nipy/heudiconv: V0.5.4: [0.5.4] - 2019-04-29. Zenodo. https://doi.org/10.5281/zenodo.2653788

Harlé, K. M., \& Sanfey, A. G. (2012). Social economic decision-making across the lifespan: An fMRI investigation. Neuropsychologia, 50(7), 1416-1424. https://doi.org/10.1016/j.neuropsychologia.2012.02.026

Hughes, C., Cassidy, B. S., Faskowitz, J., Avena-Koenigsberger, A., Sporns, O., \& Krendl, A. C. (2019). Age differences in specific neural connections within the Default Mode Network underlie theory of mind. Neurolmage, 191, 269-277. 
https://doi.org/10.1016/j.neuroimage.2019.02.024

Hughes, C., Faskowitz, J., Cassidy, B. S., Sporns, O., \& Krendl, A. C. (2020). Aging relates to a disproportionately weaker functional architecture of brain networks during rest and task states. Neurolmage, 209, 116521. https://doi.org/10.1016/j.neuroimage.2020.116521

Huxhold, O., Fiori, K. L., Webster, N. J., \& Antonucci, T. C. (2020). The Strength of Weaker Ties: An Underexplored Resource for Maintaining Emotional Well-Being in Later Life. The Journals of Gerontology: Series B, 75(7), 1433-1442.

https://doi.org/10.1093/geronb/gbaa019

Isaacowitz, D. M., Freund, A. M., Mayr, U., Rothermund, K., \& Tobler, P. N. (2021). Age-Related Changes in the Role of Social Motivation: Implications for Healthy Aging. The Journals of Gerontology: Series B, gbab032. https://doi.org/10.1093/geronb/gbab032

Jenkinson, M., Bannister, P., Brady, M., \& Smith, S. (2002). Improved Optimization for the Robust and Accurate Linear Registration and Motion Correction of Brain Images.

Neurolmage, 17(2), 825-841. https://doi.org/10.1006/nimg.2002.1132

Jenkinson, M., Beckmann, C. F., Behrens, T. E. J., Woolrich, M. W., \& Smith, S. M. (2012).

FSL. Neurolmage, 62(2), 782-790. https://doi.org/10.1016/j.neuroimage.2011.09.015

Jenkinson, M., \& Smith, S. (2001). A global optimisation method for robust affine registration of brain images. Medical Image Analysis, 5(2), 143-156. https://doi.org/10.1016/S13618415(01)00036-6

Kwak, S., Joo, W., Youm, Y., \& Chey, J. (2018). Social brain volume is associated with indegree social network size among older adults. Proceedings of the Royal Society B: Biological Sciences, 285(1871), 20172708.

Lanczos, C. (1964). Evaluation of Noisy Data. Journal of the Society for Industrial and Applied Mathematics Series B Numerical Analysis, 1(1), 76-85. https://doi.org/10.1137/0701007 Laurita, A. C., DuPre, E., Ebner, N. C., Turner, G. R., \& Spreng, R. N. (2020). Default network interactivity during mentalizing about known others is modulated by age and social 
closeness. Social Cognitive and Affective Neuroscience, 15(5), 537-549.

https://doi.org/10.1093/scan/nsaa067

Lichtenberg, P. A. (2016). Financial exploitation, financial capacity, and Alzheimer's disease.

American Psychologist, 71(4), 312.

Lichtenberg, P. A., Stickney, L., \& Paulson, D. (2013). Is psychological vulnerability related to the experience of fraud in older adults? Clinical Gerontologist, 36(2), 132-146.

Lighthall, N. R., Pearson, J. M., Huettel, S. A., \& Cabeza, R. (2018). Feedback-Based Learning in Aging: Contributions and Trajectories of Change in Striatal and Hippocampal Systems. Journal of Neuroscience, 38(39), 8453-8462. https://doi.org/10.1523/JNEUROSCI.0769-18.2018

Mars, R. B., Neubert, F.-X., Noonan, M. P., Sallet, J., Toni, I., \& Rushworth, M. F. S. (2012). On the relationship between the "default mode network" and the "social brain." Frontiers in Human Neuroscience, 6. https://doi.org/10.3389/fnhum.2012.00189

Miletić, S., Boag, R. J., \& Forstmann, B. U. (2020). Mutual benefits: Combining reinforcement learning with sequential sampling models. Neuropsychologia, 136, 107261. https://doi.org/10.1016/j.neuropsychologia.2019.107261

Nguyen, A. L., Mosqueda, L., Windisch, N., Weissberger, G., Axelrod, J., \& Han, S. D. (2021). Perceived Types, Causes, and Consequences of Financial Exploitation: Narratives From Older Adults. The Journals of Gerontology: Series B, 76(5), 996-1004.

Nickerson, L. D., Smith, S. M., Öngür, D., \& Beckmann, C. F. (2017). Using Dual Regression to Investigate Network Shape and Amplitude in Functional Connectivity Analyses. Frontiers in Neuroscience, 11. https://doi.org/10.3389/fnins.2017.00115

O'Reilly, J. X., Woolrich, M. W., Behrens, T. E. J., Smith, S. M., \& Johansen-Berg, H. (2012). Tools of the trade: Psychophysiological interactions and functional connectivity. Social Cognitive and Affective Neuroscience, 7(5), 604-609. https://doi.org/10.1093/scan/nss055 
Persson, J., Nyberg, L., Lind, J., Larsson, A., Nilsson, L.-G., Ingvar, M., \& Buckner, R. L. (2006). Structure-Function Correlates of Cognitive Decline in Aging. Cerebral Cortex, 16(7), 907-915. https://doi.org/10.1093/cercor/bhj036

Prins, N. D., \& Scheltens, P. (2015). White matter hyperintensities, cognitive impairment and dementia: An update. Nature Reviews Neurology, 11(3), 157-165.

Rademacher, L., Salama, A., Gründer, G., \& Spreckelmeyer, K. N. (2014). Differential patterns of nucleus accumbens activation during anticipation of monetary and social reward in young and older adults. Social Cognitive and Affective Neuroscience, 9(6), 825-831. https://doi.org/10.1093/scan/nst047

Samanez-Larkin, G. R., \& Knutson, B. (2015). Decision making in the ageing brain: Changes in affective and motivational circuits. Nature Reviews Neuroscience, 16(5), 278-289. https://doi.org/10.1038/nrn3917

Santini, Z. I., Koyanagi, A., Tyrovolas, S., \& Haro, J. M. (2015). The association of relationship quality and social networks with depression, anxiety, and suicidal ideation among older married adults: Findings from a cross-sectional analysis of the Irish Longitudinal Study on Ageing (TILDA). Journal of Affective Disorders, 179, 134-141.

Seaman, K. L., Brooks, N., Karrer, T. M., Castrellon, J. J., Perkins, S. F., Dang, L. C., Hsu, M., Zald, D. H., \& Samanez-Larkin, G. R. (2018). Subjective value representations during effort, probability and time discounting across adulthood. Social Cognitive and Affective Neuroscience, 13(5), 449-459. https://doi.org/10.1093/scan/nsy021

Shao, J., Zhang, Q., Ren, Y., Li, X., \& Lin, T. (2019). Why are older adults victims of fraud? Current knowledge and prospects regarding older adults' vulnerability to fraud. Journal of Elder Abuse \& Neglect, 31(3), 225-243.

https://doi.org/10.1080/08946566.2019.1625842

Smith, D. V., \& Delgado, M. R. (2017). Meta-analysis of psychophysiological interactions: Revisiting cluster-level thresholding and sample sizes. Human Brain Mapping, 38(1), 
588-591. https://doi.org/10.1002/hbm.23354

Smith, D. V., Gseir, M., Speer, M. E., \& Delgado, M. R. (2016). Toward a cumulative science of functional integration: A meta-analysis of psychophysiological interactions. Human Brain Mapping, 37(8), 2904-2917. https://doi.org/10.1002/hbm.23216

Smith, S. M., Fox, P. T., Miller, K. L., Glahn, D. C., Fox, P. M., Mackay, C. E., Filippini, N., Watkins, K. E., Toro, R., Laird, A. R., \& Beckmann, C. F. (2009). Correspondence of the brain's functional architecture during activation and rest. Proceedings of the National Academy of Sciences, 106(31), 13040-13045.

Smith, S. M., Jenkinson, M., Woolrich, M. W., Beckmann, C. F., Behrens, T. E. J., JohansenBerg, H., Bannister, P. R., De Luca, M., Drobnjak, I., Flitney, D. E., Niazy, R. K., Saunders, J., Vickers, J., Zhang, Y., De Stefano, N., Brady, J. M., \& Matthews, P. M. (2004). Advances in functional and structural MR image analysis and implementation as FSL. Neurolmage, 23, S208-S219. https://doi.org/10.1016/j.neuroimage.2004.07.051

Spreng, R. N., Cassidy, B. N., Darboh, B. S., DuPre, E., Lockrow, A. W., Setton, R., \& Turner, G. R. (2017). Financial Exploitation Is Associated With Structural and Functional Brain Differences in Healthy Older Adults. The Journals of Gerontology: Series A, 72(10), 1365-1368. https://doi.org/10.1093/gerona/glx051

Spreng, R. N., Dimas, E., Mwilambwe-Tshilobo, L., Dagher, A., Koellinger, P., Nave, G., Ong, A., Kernbach, J. M., Wiecki, T. V., Ge, T., Li, Y., Holmes, A. J., Yeo, B. T. T., Turner, G. R., Dunbar, R. I. M., \& Bzdok, D. (2020). The default network of the human brain is associated with perceived social isolation. Nature Communications, 11(1), 6393. https://doi.org/10.1038/s41467-020-20039-w

Spreng, R. N., Karlawash, J., \& Marson, D. (2016). Cognitive, social, and neural determinants of diminished decision-making and financial exploitation risk in aging and dementia: A review and new model. Journal of Elder Abuse \& Neglect, 28(4-5), 320-344. https://doi.org/10.1080/08946566.2016.1237918 
Spreng, R. N., Shoemaker, L., \& Turner, G. R. (2017). Executive functions and neurocognitive aging. In Executive functions in health and disease (pp. 169-196). Elsevier Academic Press. https://doi.org/10.1016/B978-0-12-803676-1.00008-8

Suzuki, A. (2018). Persistent Reliance on Facial Appearance Among Older Adults When Judging Someone's Trustworthiness. The Journals of Gerontology: Series B, 73(4), 573-583. https://doi.org/10.1093/geronb/gbw034

Suzuki, A., Ueno, M., Ishikawa, K., Kobayashi, A., Okubo, M., \& Nakai, T. (2019). Age-related differences in the activation of the mentalizing- and reward-related brain regions during the learning of others' true trustworthiness. Neurobiology of Aging, 73, 1-8. https://doi.org/10.1016/j.neurobiolaging.2018.09.002

Tustison, N. J., Avants, B. B., Cook, P. A., Zheng, Y., Egan, A., Yushkevich, P. A., \& Gee, J. C. (2010). N4ITK: Improved N3 Bias Correction. IEEE Transactions on Medical Imaging, 29(6), 1310-1320. https://doi.org/10.1109/TMI.2010.2046908

Uchino, B. N., \& Rook, K. S. (2020). Emotions, relationships, health and illness into old age. Maturitas, 139, 42-48. https://doi.org/10.1016/j.maturitas.2020.05.014

Utevsky, A. V., Smith, D. V., Young, J. S., \& Huettel, S. A. (2017). Large-Scale Network Coupling with the Fusiform Cortex Facilitates Future Social Motivation. ENeuro, 4(5). https://doi.org/10.1523/ENEURO.0084-17.2017

Waltz, J. A., Kasanova, Z., Ross, T. J., Salmeron, B. J., McMahon, R. P., Gold, J. M., \& Stein, E. A. (2013). The Roles of Reward, Default, and Executive Control Networks in SetShifting Impairments in Schizophrenia. PLOS ONE, 8(2), e57257. https://doi.org/10.1371/journal.pone.0057257

Webb, B., Hine, A. C., \& Bailey, P. E. (2016). Difficulty in differentiating trustworthiness from untrustworthiness in older age. Developmental Psychology, 52(6), 985. https://doi.org/10.1037/dev0000126

Weissberger, G. H., Mosqueda, L., Nguyen, A. L., Axelrod, J., Nguyen, C. P., Boyle, P. A., 
Spreng, R. N., \& Han, S. D. (2020). Functional Connectivity Correlates of Perceived

Financial Exploitation in Older Adults. Frontiers in Aging Neuroscience, 12.

https://doi.org/10.3389/fnagi.2020.583433

Woolrich, M. W., Behrens, T. E. J., Beckmann, C. F., Jenkinson, M., \& Smith, S. M. (2004).

Multilevel linear modelling for FMRI group analysis using Bayesian inference.

Neurolmage, 21(4), 1732-1747. https://doi.org/10.1016/j.neuroimage.2003.12.023

Woolrich, M. W., Ripley, B. D., Brady, M., \& Smith, S. M. (2001). Temporal Autocorrelation in Univariate Linear Modeling of FMRI Data. Neurolmage, 14(6), 1370-1386.

https://doi.org/10.1006/nimg.2001.0931

Worsley, K. J. (2001). Statistical Analysis of Activation Images. In Functional MRI: An Introduction to Methods.

Zhang, Y., Brady, M., \& Smith, S. (2001). Segmentation of brain MR images through a hidden Markov random field model and the expectation-maximization algorithm. IEEE Transactions on Medical Imaging, 20(1), 45-57. https://doi.org/10.1109/42.906424 